\title{
Analysis of azimuthal variation of rain attenuation
}

\author{
L. M. Tomaz ${ }^{1}$, C. Capsoni ${ }^{1,2}$ \\ ${ }^{1}$ Dipartimento di Elettronica, Informazione e Bioingegneria, Politecnico di Milano, Milan, Italy \\ \{1uciano.machado, carlo.capsoni\}@polimi.it. \\ ${ }^{2}$ Istituto di Elettronica e di Ingegneria dell'Informazione e delle Telecomunicazioni IEIIT, National Research Council
}

\begin{abstract}
The attenuation due to rain is the main source of impairments in satellite links operating above $10 \mathrm{GHz}$. In the design of systems based on Low Earth Orbit (LEO) satellites, the analysis of the rain attenuation must take into account both azimuth and elevation variations. It requires a deep knowledge on the nature of this dependence. As measurements at several elevation and azimuth angles are not available, it is necessary to use prediction models that aim at reproducing the rain profiles of each link. This paper presents an analysis of the azimuth dependence of rain attenuation according to the size of the azimuthal window where this attenuation occurs. It is shown that the azimuthal rain attenuation windows smaller than $180^{\circ}$ are few at higher elevation but their probability increases exponentially with the decreasing of the elevation.
\end{abstract}

Index Terms-Propagation, LEO Satellites, Rain Attenuation.

\section{INTRODUCTION}

The satellite communications technology has been developing rapidly in the last decades in order to seek increasingly larger bandwidths. It requires operating at higher frequency carriers (such as $\mathrm{Ka}$ and $\mathrm{Q} / \mathrm{V}$ bands) that allows the development of broadband systems. To make feasible the use of satellites operating at these frequencies, it is necessary to adopt Propagation Impairment Mitigation Techniques (PIMT). They consist in the detection of the propagation impairment levels and, based on them, the activation of the most efficient compensation technique and its application in real time [1].

At such high frequencies, the attenuation that arises from the effects of the atmosphere (oxygen, water vapor, clouds and precipitation particles) on the propagation path increases. Among these effects, rain is certainly the most significant source of impairment [2]. The losses are caused mainly by the raindrops absorption and scattering mechanisms. At frequencies above $10 \mathrm{GHz}$ the imaginary part of the complex permittivity of water becomes significant and then it is not possible to consider the raindrop as a lossless dielectric [3].

The correct evaluation of the effects caused by rain on the satellite links requires the knowledge on both long-term statistics and time dynamics of rain attenuation. In general, there is a lack of direct measurements of rain attenuation and this leads to the need of using prediction methods able to estimate the attenuation exceeded for the required probability level which a specific link is subjected to.

Unlike geostationary (GEO) satellites, the Low Earth Orbit (LEO) satellites moves along a large range of different elevation and azimuth angles in relation to the ground station. Thus, the knowledge of the dynamics of the rain attenuation with the variations on both - azimuth and elevation - angles is necessary. However, the correct model of this dynamics is not a trivial task because of the high spatial variability of rain.

The amount of data that a LEO satellite (in polar orbit) is required to download to ground stations (GS) is increasing rapidly. The frequency band that is currently used by Earth Observation (EO) satellites - the X band - offers a limited bandwidth. Therefore, there is an increasing demand of using the allocated frequency band at $\mathrm{Ka}$ (i.e. from 25.5 to $27 \mathrm{GHz}$ ) in order to enlarge the available bandwidth by a factor of four. The lack of models able to reproduce the patterns of rain attenuation in the LEO satellite links is an obstacle to the development of tools able to mitigate the atmospheric propagation effects.

This paper analyses the dynamics of rain attenuation according to the variations in the azimuth. The analysis relies on a synthetic rain maps database obtained by the MultiEXCELL model. The paper is organized as follows: section II describes the database used and the method applied to retrieve the attenuation; section III presents the layout in which the simulations were performed; Section IV shows the preliminary results of the analysis; finally, section $\mathrm{V}$ draws some conclusions.

\section{EVAlUAtion OF RAIN AtTENUATION}

\section{A. Database}

The database used in this work is constituted by rain maps $230 \mathrm{~km} \times 230 \mathrm{~km}$ in size generated by the propagation-oriented MultiEXCELL rain field model described in [4]. MultiEXCELL was largely validated in several propagationoriented applications ( [5], [6] and [7]). It enables the generation of realistic rain fields composed by aggregates of synthetic rain cells with the exponential profile described in (1).

$$
R(\rho)=R_{M} \mathrm{e}^{-\left(\frac{\rho}{\rho_{0}}\right)^{\frac{2}{k}}},
$$

where $R$ is the rain rate in $\mathrm{mm} / \mathrm{h}, \rho$ is the distance from the cell center, $R_{M}$ is the maximum rain rate of the cell and $\rho_{0}$ is the equivalent radius for which $R\left(\rho_{0}\right)=R_{M} / e$. Finally, $k$ is the shape factor (equal to 2 for the exponential profile) [5].

The MultiEXCELL model considers that rain cells tend to aggregate into larger rain structures. This ensemble of rain cells is defined as the area with the rain rate exceeding a specified level $(1 \mathrm{~mm} / \mathrm{h})$ and having inside two or more peaks greater than $5 \mathrm{~mm} / \mathrm{h}$. More than one aggregate is present in a 
rain map. This complex description of the rain field is particularly important for the evaluation of non-geostationary links performance, in which the path length across rain becomes longer and longer with the decreasing of the elevation angle. Thus, the probability that more than one cell simultaneously interacts with the link increases strongly.

In this work, we used 578 synthetic rain maps generated by MultiEXCELL whose ensemble reproduces the local rain intensity statistics and maintain the spatial distribution of rain in a specific place. The model uses as input the annual cumulative distribution of rain intensity for the selected site. Fig. 1 shows a sample rain map provided by the model.

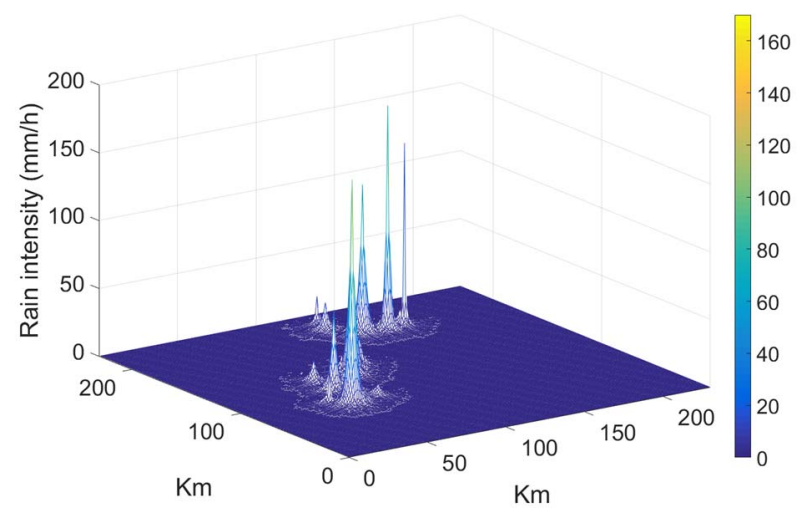

Fig. 1: Example of rain map generated by the MultiEXCELL model.

For this preliminary study, the maps refer to the site Spino D'Adda, Italy $\left(45.4^{\circ} \mathrm{N}, 9.5^{\circ} \mathrm{E}\right)$. Each map covers an area of $52900 \mathrm{~km}^{2}$ and the spatial resolution is $1 \mathrm{~km}^{2}$.

\section{B. Evaluation of rain attenuation from the rain rate database}

The conversion between rain rate and rain attenuation depends on the link parameters (frequency, polarization, elevation angle, etc.) and can be easily achieved using (2) [8].

$$
A(d B)=\int_{0}^{L_{s}} \kappa R^{\alpha} d L,
$$

where $L_{s}$ is the effective slant-path length across the rain in $\mathrm{km}$ that depends on the elevation angle and the rain height the difference between the mean annual $0^{\circ} \mathrm{C}$ isotherm height above mean sea level and the height of the site - and can be retrieved by the procedure proposed in ITU-R Recommendation P.839 [9], $R$ is the rain intensity spatially distributed according to the synthetic rain maps of the database. The parameters $\kappa$ and $\alpha$ are dependent on frequency, polarization and elevation angle, and are also function of the Drop Size Distribution (DSD), as well as of the refractivity index of water which, in turn, depends on the temperature of water. These parameters can be evaluated with the procedure presented in recommendation P.838-3 [11]. The power law $\kappa R^{\alpha}$ is referred to as the specific attenuation $(\gamma)$ and is given in $\mathrm{dB} / \mathrm{km}$.
Aiming to obtain a statistical significant number of samples, the simulations were performed by moving the ground station position pixel by pixel along the whole map and repeating this procedure for each of the 578 maps.

There are cases where the horizontal projection of $L_{s}-$ in the integration procedure presented in (2) - crosses the boundary of the map: it is then necessary to extend the rain field. This is done by placing a second map alongside the first one does this.

We assume that the resulting larger area has approximately the same rain coverage, which is acceptable when considering no big changes in the orography. The preliminary solution adopted here is the use of a rotated version of the first map to enlarge the rain field. This procedure is necessary for the analysis of low elevation angles. In this case, most of the links exceed the size of the first map.

\section{SiMULATIONS}

MultiEXCELL was already validated in several propagation-oriented applications. Using the procedure described in Section II, we performed simulations to estimate the rain attenuation for several azimuth angles $\left(0^{\circ}\right.$ to $179^{\circ}$ in $1^{\circ}$ step $)$ and seven different elevations $\left(20^{\circ}, 37^{\circ}, 45^{\circ}, 60^{\circ}, 80^{\circ}\right.$ and $90^{\circ}$ ). The frequency used was $39.6 \mathrm{GHz}$ with circular polarization. The choice of this frequency in this preliminary stage of the study is due to fact that a reference CCDF of rain attenuation was available for Spino D'Adda and it was used to validate the procedure. Fig. 2 illustrates the simulation procedure.

The radius (L) of each circle - equivalent to the horizontal projection of the path length - is dependent on the elevation angle and the rain height. For Spino D'Adda, we consider a rain height of $2.9 \mathrm{~km}$, which gives an effective path length ranging from, e.g., $2.94 \mathrm{~km}$ (at $80^{\circ}$ elevation) to around 8.48 $\mathrm{km}$ (at $20^{\circ}$ elevation).

From Fig. 2 it is easy to identify three possible situations of the link position with respect to the rain structure. The situations can be divided as follows:

\section{A. Rainy azimuth window $=180^{\circ}$}

It is clear that in all cases when the ground station is inside the rain structure, the rain attenuation is extending over an azimuth window of $180^{\circ}$.

\section{B. Rainy azimuth window $<180^{\circ}$}

Conversely, if the circles identified by the horizontal projection of the link cross the rain structure but the ground station is outside, the azimuthal rain attenuation window assumes values between $1^{\circ}$ and $180^{\circ}$.

\section{No rain attenuation}

Finally, if the circle is completely outside the rain structure, there will be no rain attenuation.

\section{PRELIMINARY RESULTS}

According to [3], when the terrain is flat over a large area around the site, the attenuation levels for different azimuth 
angles will not show strong variations. Fig. 3 shows the simulated annual CCDF of rain attenuation for Spino D'Adda at three different azimuth angles $\left(0^{\circ}, 30^{\circ}\right.$ and $\left.60^{\circ}\right)$ for an elevation of $37^{\circ}$.

Based on the simulations it was possible to identify the probabilities of occurrence for each of the situations described in section III. Over a total of 30576200 (230x230x578) samples per azimuth, it is evaluated the size (in degrees) of the azimuthal window for which the attenuation is greater than zero. The procedure is the following:

1. For each pixel of a map a vector with 180 positions is stored containing the samples at all the azimuth angles (from $0^{\circ}$ to $179^{\circ}$ );

2. Then we count the number of non-zeros contiguous samples in each vector from which to derive the size of the azimuthal rain attenuation window as shown in Fig. 4.

Table 1 presents the probabilities of the three possible situations for all the mentioned elevations angle. The probability of having an azimuth rain attenuation window equal to $180^{\circ}$ is almost constant because it is equivalent to the number of rainy pixels in the maps. The small fluctuations in the values of this probability is due the fact that we have performed simulations just from $0^{\circ}$ to $179^{\circ}$ and not a complete $360^{\circ}$ rotation.

On the other hand, the probability of having an azimuth rain attenuation window smaller than $180^{\circ}$ is highly dependent on the link elevation angle and to a lesser extent on the site (rain height and rain patterns - stratiform or convective).

This probability is exponentially related to the elevation angle following (3).

$$
p\left(a z_{w}<180^{\circ}\right)=0.1294 e^{-0.0346 \theta}
$$

Where $p\left(\mathrm{az}_{\mathrm{w}}<180^{\circ}\right)$ is the probability of having an azimuthal rain attenuation window smaller than $180^{\circ}$ and $\theta$ is the elevation angle in degrees. Fig. 5 shows the exponential relation with the data obtained from the simulations.

\section{TABLE 1: PROBABILITIES FOR THE POSSIBLE SITUATIONS} PRESENTED IN III.

\begin{tabular}{|c|c|c|c|}
\hline Situations & & & \\
\hline Probability & III.A & III.B & III.C \\
\hline $\boldsymbol{\theta}=\mathbf{2 0}^{\circ}$ & $13.54 \%$ & $6.48 \%$ & $79.98 \%$ \\
\hline $\boldsymbol{\theta}=\mathbf{3 7}^{\circ}$ & $13.54 \%$ & $3.6 \%$ & $82.86 \%$ \\
\hline $\boldsymbol{\theta}=\mathbf{4 5}^{\circ}$ & $13.54 \%$ & $2.61 \%$ & $83.85 \%$ \\
\hline $\boldsymbol{\theta}=\mathbf{6 0}^{\circ}$ & $13.51 \%$ & $1.85 \%$ & $84.63 \%$ \\
\hline $\boldsymbol{\theta}=\mathbf{8 0}^{\circ}$ & $13.31 \%$ & $1.15 \%$ & $85.55 \%$ \\
\hline $\mathbf{9 0}^{\circ}$ & $13.05 \%$ & $0 \%$ & $86.95 \%$ \\
\hline
\end{tabular}

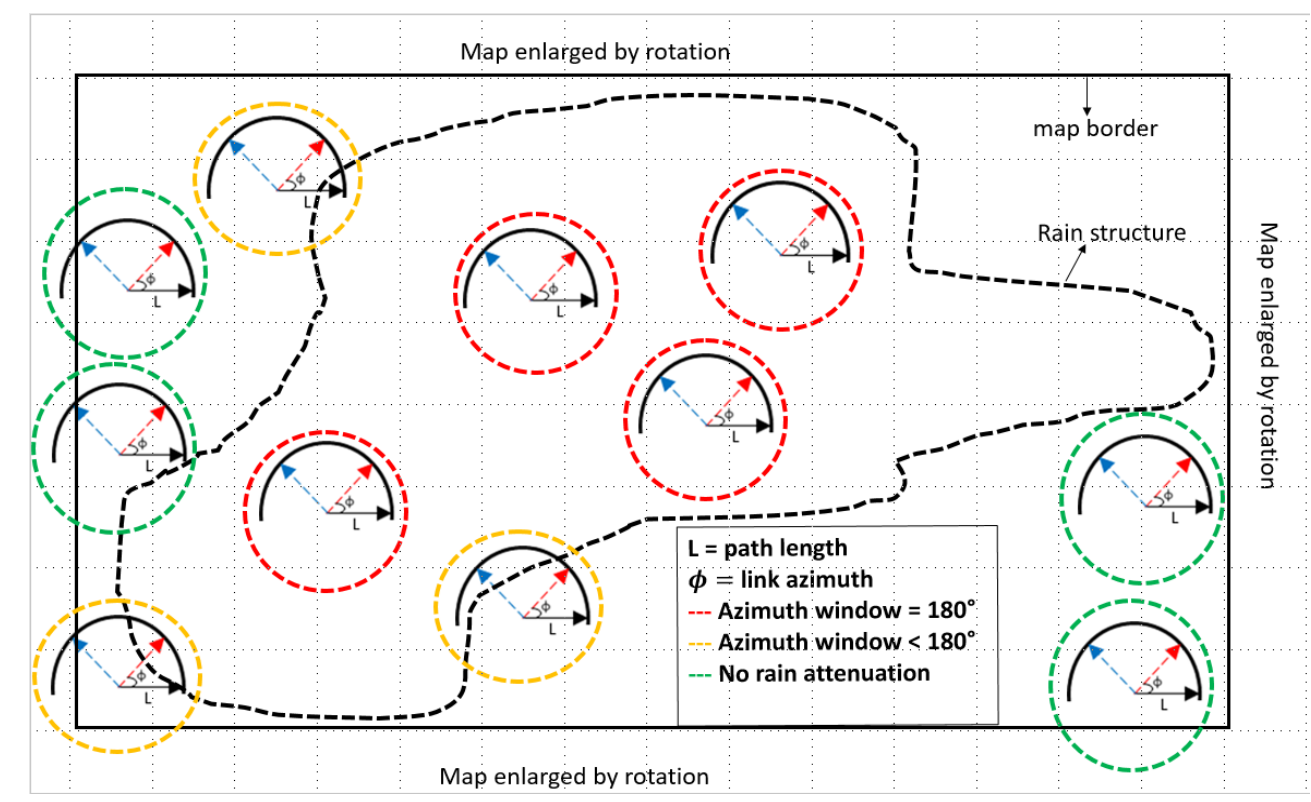

Fig. 2: Illustration of the procedure used to collect the rain attenuation samples. 


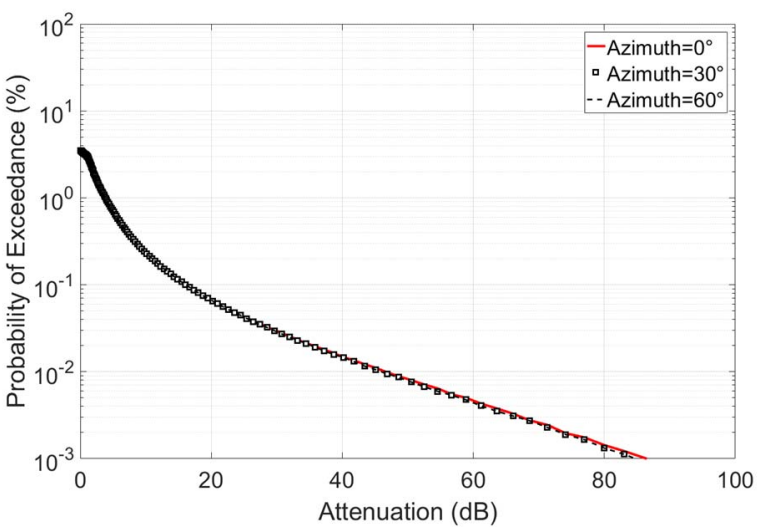

Fig. 3: Annual CCDF for different azimuth angles $(39.6 \mathrm{GHz}$, circular polarization, $37^{\circ}$ elevation)

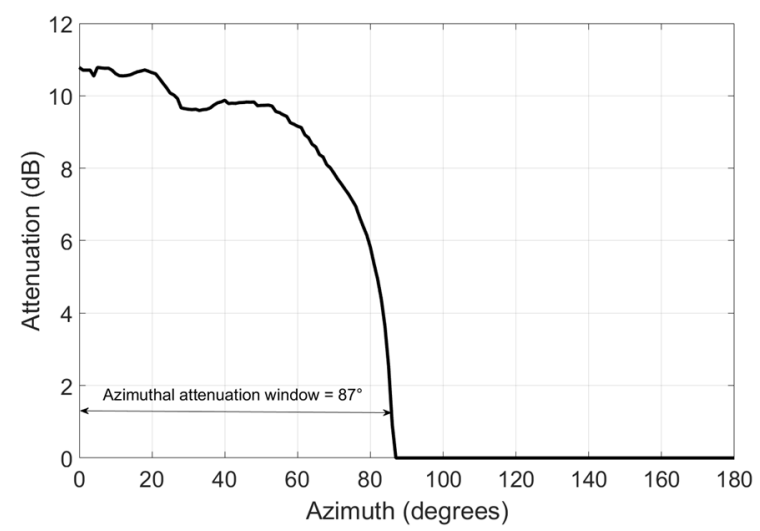

Fig. 4: Example of azimuthal attenuation window at $20^{\circ}$ elevation.

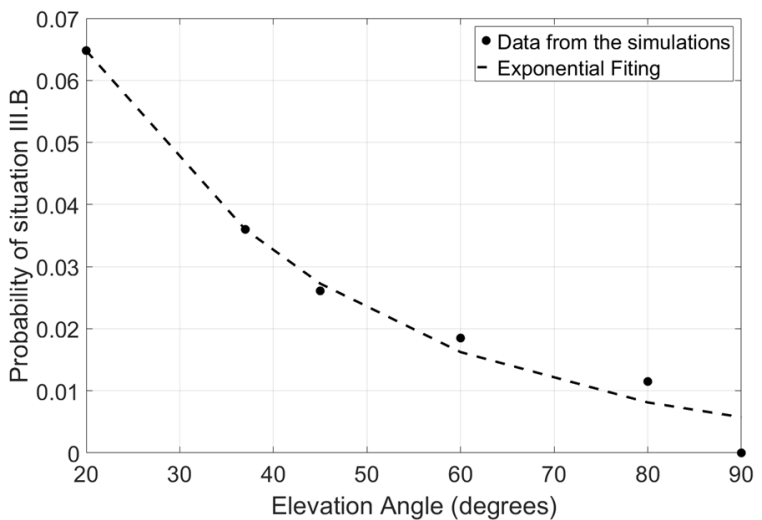

Fig. 5: Probability of situation III.B in function of the elevation angle.

Fig. 6 shows the histograms of the windows for four elevation angles. It can be seen that the number of samples has a significant increase for the lower elevations.
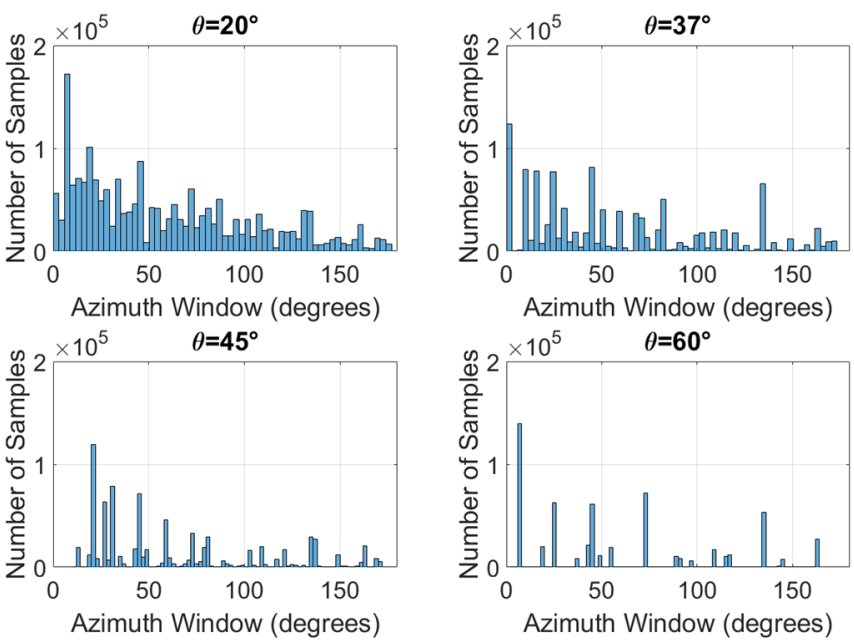

Fig. 6: Histograms for the situation describe in III.B at different elevation angles.

\section{CONCLUSION}

In this paper, we presented an analysis of the dynamics of rain attenuation according to the variations in azimuth for satellite links. This was done in terms of the size of the azimuthal window in which the rain attenuation is greater than zero. The percentage of azimuthal windows below $180^{\circ}$ degrees increases exponentially with the decreasing of elevation. It was also derived an exponential equation for this relation. This analysis can be useful especially in the case of the LEO satellite links in which elevation and azimuth positions are constantly changing in time.

\section{ACKNOWLEDGMENT}

This work was partially supported by $\mathrm{CNPq}$ (Conselho Nacional de Desenvolvimento Científico e Tecnológico Brazilian research support institution). The authors would like to acknowledge the many valuable suggestions made by Lorenzo Luini who also provides us with the rain maps used in this work.

\section{REFERENCES}

[1] F. Lacoste, G. Carrie and C. L. Castanet, "Validation of rain attenuation time series synthesizers for temperate area - on the enhanced Maseng-Bakken model," in IEEE International Workshop on Satellite and Space Communications, 2008.

[2] A. K. Maini and V. Agrawal, Satellite technology : principles and applications, Chichester: John Wiley \& Sons, 2014.

[3] J. E. Allnutt, Satellite-to-Grund Radiowave Propagation, London: The Institution of Engineering and Technology, 2011.

[4] L. Luini and C. Capsoni, "MultiEXCELL: A new rain field model for propagation applications," IEEE Transactions on Antennas and Propagation, November 2011.

[5] L. Luini and C. Capsoni, "A rain cell model for the simulation and performance evaluation of site diversity schemes," vol. 12, pp. 1327$1330,2013$.

[6] L. Luini and C. Capsoni, "A physically based methodology for the evaluation of the rain attenuation on terrestrial radio links," in EuCAP, Barcelona, 2010. 
[7] C. Capsoni, L. Luini and M. D'Amico, "The MultiEXCELL model for the prediction of the radio interference due to hydrometeor scattering," in EuCAP, Barcelona, 2010.

[8] L. Ippolito, Radiowave propagation in satellite communications, New York: Van Nostrand Reinhold Comapany Inc., 1986.

[9] ITU-R, "Recommendation P.839-4 - 'Rain height model for prediction methods'," 2013.

[10] ITU-R, "Recommendation P.618-12: 'Propagation data and prediction methods required for the design of Earth-space telecommunication systems'," 2015.

[11] ITU-R, "Recommendation P.838-3: 'Specific attenuation model for rain for use in prediction methods'," 2005. 